\title{
A PESCA COMERCIAL de PEIXES PELÁGICOS NO ARQUIPÉlAGO de SÃo PEDRO e SÃO PAULO, BRASIL.
}

\author{
Teodoro VASKE Jr. ${ }^{1}$ \\ Rosangela Paula Teixeira LESSA ${ }^{1}$ \\ Aurelyanna Bezerra Christine RIBEIRO ${ }^{1}$ \\ Marcelo Francisco de NÓBREGA ${ }^{2}$ \\ Arley de Andrade PEREIRA ${ }^{3}$ \\ Carla Danielle Pereira de ANDRADE ${ }^{4}$
}

\begin{tabular}{l|l|}
\hline Recebido em: / / \\
\hline Aceito em: / /
\end{tabular}

Com o objetivo de se conhecer as principais espécies de peixes de valor comercial capturados no ASPSP e determinar a estrutura populacional por tamanho, foi realizada a compilação dos dados obtidos no período de 1998 e 2004. Os resultados demonstram que a atividade pesqueira no arquipélago incide sobre treze espécies de peixes, com destaque para a cavala-empinge (Acanthocybium solandri), albacora-laje (Thunnus albacares) e peixe-rei (Elagatis bipinnlata). No entanto há uma captura complementar dirigida ao voador-holandês (Cypselurus cyanopterus) e também para espécies ocasionais como o xaréu-preto (Caranx lugubris). Com relação ao impacto pesqueiro sobre as espécies capturadas foi constatado que apenas a albacora-laje esta sujeita a sobrepesca, devido à pressão pesqueira sofrida por esta espécie em todo Atlântico tropical.

Palavras chaves: Pesca, Arquipélago de São Pedro e São Paulo,

\section{THE COMMERCIAL FISHERY OF PELAGIC FISHES OF THE SAINT PETER AND SAINT PAUL ARCHIPELAGO, BRASIL.}

\begin{abstract}
The aim of this study was to describe the main commercial fishes captured by local fishery in the Saint Peter and Saint Paul Archipelago, and determine the structure population in size, between 1998 and 2004. Results have demonstrated that fishing activities in the archipelago was performed upon three main species, wahoo (Acanthocybium solandri), yellowfin tuna (Thunnus albacares), and the rainbow runner (Elagatis bipinnulata). Nevertheless, there is a complementar capture of margined flying fish (Cypselurus cyanopterus), and also occasional catches of black jack (Caranx lugubris). The fishery impact is remarkable only in the yellowfin tuna population, that is consequence of the fishery pressure performed in the whole tropical Atlantic.
\end{abstract}

Keywords: fishery. Saint Peter and Saint Paul Archipelago

\section{INTRODUÇÃo}

As atividades de pesca visando atuns e afins no nordeste do Brasil tiveram início no final dos anos cinqüenta, com o arrendamento de embarcações japonesas operando a partir do Porto de Recife-PE. A partir de 1977, trabalhos de prospecção tiveram início no Arquipélago de São Pedro e São Paulo (ASPSP), com campanhas em 1977/78 pelo BPq Diadorim (IBAMA) utilizando pesca do corrico, rede de espera e linha de mão, e em 1985/86, pelo NPq Riobaldo (IBAMA) utilizando corrico, linha de mão de superfície e de fundo, e puçá (Oliveira et al., 1997; Evangelista et al., 1998). Em 1988, embarcações sediadas em Natal-RN iniciaram atividades no ASPSP visando uma pesca direcionada principalmente para a albacora-laje (Thunnus albacares), cavala-empinge (Acanthocybium solandri) e voador-holandês (Cypselurus cyanopterus) (Oliveira et al, 1997; Hazin, 1998). Posteriormente houve a realização de uma exploração pesqueira científica pelo NPq Riobaldo (Projeto ECOTUNA) entre 1992 e 1995. E em 1998 foi implantada a Estação

\footnotetext{
Contantos: ${ }^{1}$ Laboratório de Dinâmica de Populações Marinhas - DIMAR/DEPAq/UFRPE.

${ }^{2}$ Fundação Universidade do Rio Grande/Programa de Pós-Graduação em Oceanografia Biológica

3 Universidade Federal de Pernambuco/Programa de Pós-Graduação em Oceanografia.

${ }^{4}$ Engenheira de Pesca/ UFRPE
} 
VASKE Jr et al. A pesca comercial de peixes pelágicos no arquipélago de São Pedro e São Paulo, Brasil.

Científica do ASPSP (ECASPSP) pela Secretaria da Comissão Interministerial para os Recursos do Mar - SECIRM que desde então, vem auxiliando em trabalhos de pesquisa sobre a pesca e biologia dos peixes do ASPSP.

Com a finalidade de se conhecer as principais espécies de peixes de valor comercial capturados no ASPSP e suas adjacências e de determinar a estrutura populacional por tamanho dessas espécies, foi feita uma compilação dos resultados de captura comercial no ASPSP entre 1998 e 2004.

\section{MATERIAL E MÉTODOS}

A pesca no ASPSP é realizada desde poucos metros dos rochedos até um raio de 10 milhas ao redor do arquipélago (Fig. 1). São utilizadas embarcações de aproximadamente 15 m de comprimento, que operam com espinhel pelágico, linha de mão, corrico e puçá.

O espinhel pelágico monofilamento de 500 anzóis é utilizado nas adjacências do ASPSP num raio de 10 milhas visando tunídeos, peixes de bico e tubarões. A pesca de corrico é realizada ao redor do arquipélago num raio máximo de 1 milha com 5 linhas preparadas com anzol MUSTAD n.o 611, rebocadas pela embarcação, e iscadas com filé de voador-holandês ou pele de tubarão cortada em formato de peixe. O corrico é realizado ao amanhecer, no início da tarde e no anoitecer, num período de uma aproximadamente uma hora. A pesca de linha de mão utiliza uma linha de náilon monofilamento de 1,8 a 2,0 mm de espessura, com anzol n.o 615, 616 ou 617, iscada com peixe-voador vivo e é realizada entre o entardecer e o amanhecer. Os puçás utilizados na pesca são confeccionados com um aro de metal de $50 \mathrm{~cm}$ de diâmetro recoberto por uma rede com malhas de 1,0 ou 2,0 $\mathrm{cm}$ fixado em um cabo de bambu com 3 a 5 metros de comprimento.

A bordo dos barcos pesqueiros que atuam na área do ASPSP, os peixes capturados foram identificados e os comprimentos zoológicos medidos em centímetros com trena. Especificamente para o voador-holandês foram obtidos dados de sexo, que por sua vez foram submetidos a um teste de Kruskall-Wallis ao nível de significância de 0,05 para verificar a existência de diferenças significativas entre os tamanhos de machos e fêmeas (Sokal \& Rohlf, 1981).

\section{RESULTADOS}

Entre junho de 1998 e junho de 2004, 4504 peixes tiveram seus comprimentos totais e zoológicos medidos, o que permitiu ter uma idéia da composição das capturas e estrutura de tamanhos das principais espécies capturadas pela pesca ao redor do ASPSP. Das 116 espécies de peixes do ASPSP (Vaske et al., 2005), treze espécies foram identificadas como tendo valor comercial.

Em torno de $99 \%$ das capturas com corrico são de cavala-empinge, com capturas muito raras de algum exemplar de xaréu (Caranx spp.), atum (Thunnus spp.) ou dourado (Coryphaena hippurus). As demais espécies foram capturadas com espinhel pelágico e linha de mão, onde ocorreram também, em menores proporções, o espadarte (Xiphias gladius), dourado e alguns tubarões com destaque para o lombo-preto (Carcharhinus falciformis) e o martelo (Sphyrna lewini) (Tab. 1). Ressalta-se ainda algumas capturas ocasionais e dirigidas ao peixe-prego (Ruvettus pretiosus), com linha de mão a profundidades superiores a $120 \mathrm{~m}$. Cinco exemplares analisados mediram entre 110 e $147 \mathrm{~cm}$ de comprimento zoológico.

As distribuições de freqüências de comprimento para as oito principais espécies de valor comercial são apresentadas para sexos agrupados na figura 2 .

Entre os atuns, os comprimentos médios de $T$. albacares e $T$. obesus foram muito próximos com 99,6 e 98,2 cm respectivamente, no entanto, o comprimento máximo para $T$. obesus foi de $184 \mathrm{~cm}$ e $175 \mathrm{~cm}$ para T. albacares. Para o voador-holandês foram analisados 1495 exemplares sendo 1160 machos e 335 fêmeas, que mostraram diferenças significativas entre os tamanhos (Kruskal-Wallis, $p=0,001$ ). Os machos foram menores com amplitudes entre 23 e 34 cm, e as fêmeas entre 26 e 39 cm (Fig. 3).

Entre as três principais espécies, albacora-laje, cavala-empinge e peixe-rei, houve uma tendência de maior produção de albacora-laje e peixe-rei entre os meses de novembro 
e março, ao passo que a produção da cavala-empinge oscilou com quantidades irregulares ao longo do ano (Fig. 4).

\section{DISCUSSÃo}

Pelo fato da pesca no ASPSP ser realizada utilizando-se diferentes artes de pesca e com capturas variáveis ao longo do ano, não é possível padronizar as capturas e os esforços, no entanto, pode-se dizer que $92,49 \%$ do total das capturas está representado pela albacora-laje, cavala-empinge, peixe-rei e voador-holandês.

A pesca da albacora-laje é atividade tradicional no ASPSP desde o início das pescarias de tunídeos no Atlântico central e ainda mantém um nível de captura bem mais alto, em torno de 3,62 peixes/ 100 anzóis, do que em áreas oceânicas adjacentes onde os valores oscilam entre 0,35 e 1,35 peixes/ 100 anzóis na pesca com espinhel (Hazin, 1993). Se for considerada apenas a pesca com linha de mão nas proximidades dos rochedos, as capturas são praticamente apenas de albacora-laje, com raras ocorrências de outros tunídeos. Ao se considerar a primeira maturação sexual de $140 \mathrm{~cm}$ (Albaret, 1977) para o Atlântico equatorial, conclui-se que a quase totalidade dos exemplares capturados no ASPSP ainda está imatura.

Oxenford (2003) realizou um levantamento dos dados de diversos autores sobre o tamanho de primeira maturação para a cavala-empinge no Atlântico oeste central e o tamanho determinado ficou entre $85 \mathrm{~cm}$ e $102 \mathrm{~cm}$. Pinheiro (2004) determinou para o peixe-rei no Arquipélago de Fernando de Noronha um tamanho médio de primeira maturação sexual de $130 \mathrm{~cm}$. A julgar pelo comprimento médio da cavalas-empinge (130 $\mathrm{cm}$ ) e do peixe-rei $(70 \mathrm{~cm})$, pode-se inferir que apesar da intensa captura no ASPSP, as populações dessas duas espécies conseguem manter o padrão de tamanhos com contínuas reposições de exemplares mais velhos que se concentram nas proximidades vindos de oceano aberto para se alimentar. Devido a essa concentração de cavala-empinge nas adjacências do ASPSP, a captura passa a ser dez vezes maior do que a obtida pela pesca de espinhel onde a cavala-empinge representou em torno de 3,7 \% entre 1992 e 2000 na ZEE nordetse do Brasil (Travassos, 2002).

A captura do tubarão lombo-preto também pode ser considerada alta no ASPSP $(2,14$ $\%$ do total), uma vez que em oceano aberto os tubarões do gênero Carcharhinus representam cerca de 0,6\% em número do total capturado (Hazin, 1993, Travassos, 2002). De acordo com Oliveira et al. (2004) em exemplares de C. falciformis analisados no ASPSP, foram encontrados todos os estágios de maturação inclusive embriões em pré-parto, portanto é provável que haja parto no local. Em estômagos de lombo-preto também foram encontrados voadores-holandeses, até 11 exemplares em um só estômago, evidenciando que o lombo-preto também está na área para se alimentar de voador-holandês.

A pesca do xaréu-preto é feita com linha normalmente no amanhecer e anoitecer ao lado das pedras, capturando alguns exemplares com objetivo apenas de complementar a pesca sendo levados por pescadores para vendas a varejo em Natal.

A pesca com puçá captura exclusivamente o voador-holandês, os exemplares capturados nessa pescaria são utilizados como isca na pesca de corrico e também servem para complementar espaços nas urnas dos barcos que não foram preenchidos pelos grandes peixes de valor comercial. O voador-holandês ocorre ao longo de todo ano, no entanto as maiores concentrações ocorrem entre novembro e março que correspondem ao seu pico de período reprodutivo no local (Lessa et al., 1999), e é por esse fato que também se explica as maiores capturas de albacora-laje e peixe-rei entre novembro e março, uma vez que a concentração desses predadores nesse período ocorre em virtude da predação sobre os densos cardumes de voador-holandês (Lessa et al., 1999; Vaske Jr. et al., 2003).

Em relação ao impacto pesqueiro nas espécies capturadas no ASPSP pode-se concluir que apenas a albacora-laje está sujeita a sobrepesca, em virtude da pressão pesqueira que esta espécie sofre em todo Atlântico tropical, onde o rendimento máximo sustentável está no limite em torno de 150.000 t (ICCAT, 2003; Lessa et al., 2004) e que deve ter implicações nos pequenos tamanhos de exemplares capturados no ASPSP. Os tamanhos de captura de cavala-empinge, peixe-rei, xaréu-preto e voador-holandês revelam maturidade 
VASKE Jr et al. A pesca comercial de peixes pelágicos no arquipélago de São Pedro e São Paulo, Brasil.

dos exemplares, e os níveis de captura atuais podem ser mantidos graças a pequena pressão pesqueira que a frota local exerce sobre os cardumes no ASPSP.

\section{BIBLIOGRAFIA}

ALBARET, J. J. La reproducción de l'albacore (Thunnus albacares) dans le Golfe de Guinée. Cah. ORSTOM (Sér. Océanogr.) 15 (4): 389-419. 1977

EVANGELISTA, J. V.; OLIVEIRA. G. M.; VASCONCELOS, J. A. Evolução da pesca de atuns no nordeste do Brasil. Bol. Técnico-Científico do CEPENE , vol.6 (1): 77-108. 1998.

HAZIN, F. H. V. Fisheries-oceanographical study on tunas, billfishes and sharks in the southwestern equatorial Atlantic Ocean. Tóquio 1993. 286 f. Tese (Doutorado). Universidade de Pesca de Tóquio, Japão.

HAZIN, F. H. V.1998. Programa REVIZEE-SECIRM. Levantamento de dados pretéritos Grandes peixes pelágicos do nordeste (atuns, agulhões e tubarões). Recife. Manuscrito,46 p.

HAZIN, F. H.; A.A. COUTO; K. KIHARA; K. OTSUKA; M. ISHINO. Distribution and abundance of pelagic sharks in the southwestern equatorial Atlantic. J. Tokio Univ. Fish. vol 77(1):51-64. 1990

ICCAT, Report of the standing committee on research \& statistic. SCRS. ICCAT report II, 201 p. 2003

LESSA, R. P. T; MAFALDA-JR, P; ADVÍNCULA. R.; LUCCHESI, R; BEZERRA-JR., J. L; VASKE-JR, T.; HELLEBRANDT, D. Distribution and abundance of ichthyoneuston at seamounts and islands off north-eastern Brazil. Arch. Fish. Res. 47 (2/3):133-146. 1999 a

LESSA, R. P. T.; NÓBREGA, M. F.; BEZERRA-JR, J. L. Dinâmica de Populações e Avaliação de Estoques dos Recursos Pesqueiros da Região Nordeste. REVIZEE SCORE-NE. Vol. 2. 231 p. 2004.

OLIVEIRA, G. M..; EVANGELISTA, J. E. V.; FERREIRA, B. P. Considerações sobre a biologia e a pesca no Arquipélago dos Penedos São Pedro e São Paulo. Bol. Técnico-Científico do CEPENE. vol.5 (1):31-52. 1997.

OLIVEIRA, P. G. V.; HAZIN, F. H. V.; VÉRAS, D; FISCHER, A; LUCENA, B. C. L.; CARVALHO, F.; AMORIM, M; VIANA, D. Dados preliminares acerca da biologia reprodutiva dos tubarões lombo-preto, Carcharhinus falciformis (Bibron, 1839) capturados no ASPSP. RESUMOS DA IV REUNIÃ̃o DA SBEEL, João Pessoa - PB, Resumo João Pessoa - PB 2004.

OXENFORD, H. A.; MURRAY, P. A.; LUCKHURST, B. L. The biology of wahoo (Acanthocybium solandri) in the Western Central Atlantic. Gulf and Caribbean Research, 15: 33-49. 2003

PINHEIRO, P.B. Biologia reprodutiva do peixe-rei, Elagatis bipinnulata (Quoy \& Gaimard, 1825), capturado na Zona Econômica Exclusiva (ZEE) do nordeste do Brasil. Recife, 2004. 58 f. Dissertação (Mestrado em Oceanografia). Departamento de Oceanografia Universidade Federal de Pernambuco.

SOKAL, R. R \& ROHLF, F. J. Biometry. The principles and practice of statistics in biological research. New York: W. H. Freeman \& Co. 1981. 859p.

TRAVASSOS, P. E. Variabilidade das condições climáticas e oceanográficas e suas influências sobre a distribuição e capturabilidade das principais espécies pelágicas do Atlântico oeste tropical. CNPq. 85 p. Relatório final de bolsa de Desenvolvimento Científico Regional/CNPq. 2002.

VASKE JR., T. ; C.M. VOOREN \& R.P. LESSA. Feeding habits of yellowfin tuna (Thunnus albacares), and wahoo (Acanthocybium solandri) in the Saint Peter and Saint Paul Archipelago, Brazil. Bol. Inst. Pesca 29 (1):173-181. 2003

VASKE JR., T.; R. P. LESSA; M.F. NÓBREGA; S. MONTEALEGRE-QUIJANO; F.M. SANTANA; J.L. BEZERRA JR.. A checklist of fishes from Saint Peter and Saint Paul Archipelago, Brazil. J. Applied Ichthyology 21 (1):75-79. 2005. 
Tropical Oceanography, Recife, v. 36, n. 1-2, p. 47-54, 2008.

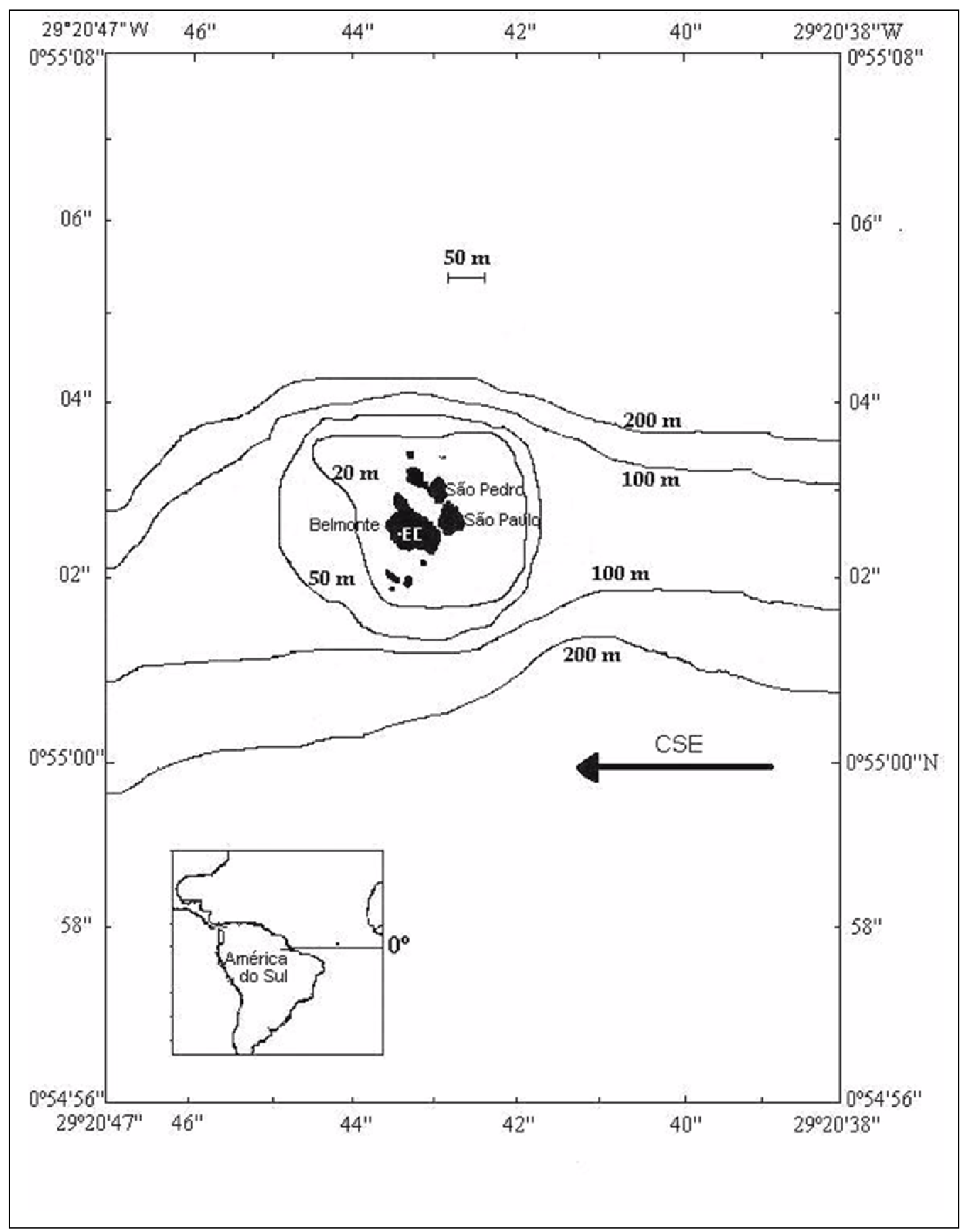

Figura 1 - Área de pesca no Arquipélago de São Pedro e São Paulo com isóbatas. CSE Corrente Sul Equatorial. 
VASKE Jr et al. A pesca comercial de peixes pelágicos no arquipélago de São Pedro e São Paulo, Brasil.

Tabela 1 - Peixes analisados oriundos da frota comercial no Arquipélago de São Pedro e São Paulo com as respectivas capturas absolutas e relativas.

\begin{tabular}{llrr}
\hline Espécie & Nome comum & Número & \% \\
\hline Cypselurus cyanopterus & Voador-holandês & 1495 & 33,19 \\
Acanthocybium solandri & Cavala-empinge & 1131 & 25,11 \\
Thunnus albacares & Albacora-laje & 1010 & 22,42 \\
Elagatis bipinnulata & Peixe-rei & 530 & 11,77 \\
Caranx lugubris & Xaréu-preto & 109 & 2,42 \\
Thunnus obesus & Albacora-bandolim & 79 & 1,75 \\
Carcharhinus falciformis Lombo-preto & 63 & 1,40 \\
Coryphaena hippurus & Dourado & 26 & 0,58 \\
Xiphias gladius & Espadarte & 25 & 0,56 \\
Prionace glauca & Tubarão-azul & 20 & 0,44 \\
Sphyrna lewini & Tubarão-martelo & 6 & 0,13 \\
Ruvettus pretiosus & Peixe-prego & 5 & 0,11 \\
Thunnus alalunga & Albacora-branca & 5 & 0,11 \\
\hline TOTAL & & $\mathbf{4 5 0 4}$ & $\mathbf{1 0 0}$ \\
\hline
\end{tabular}



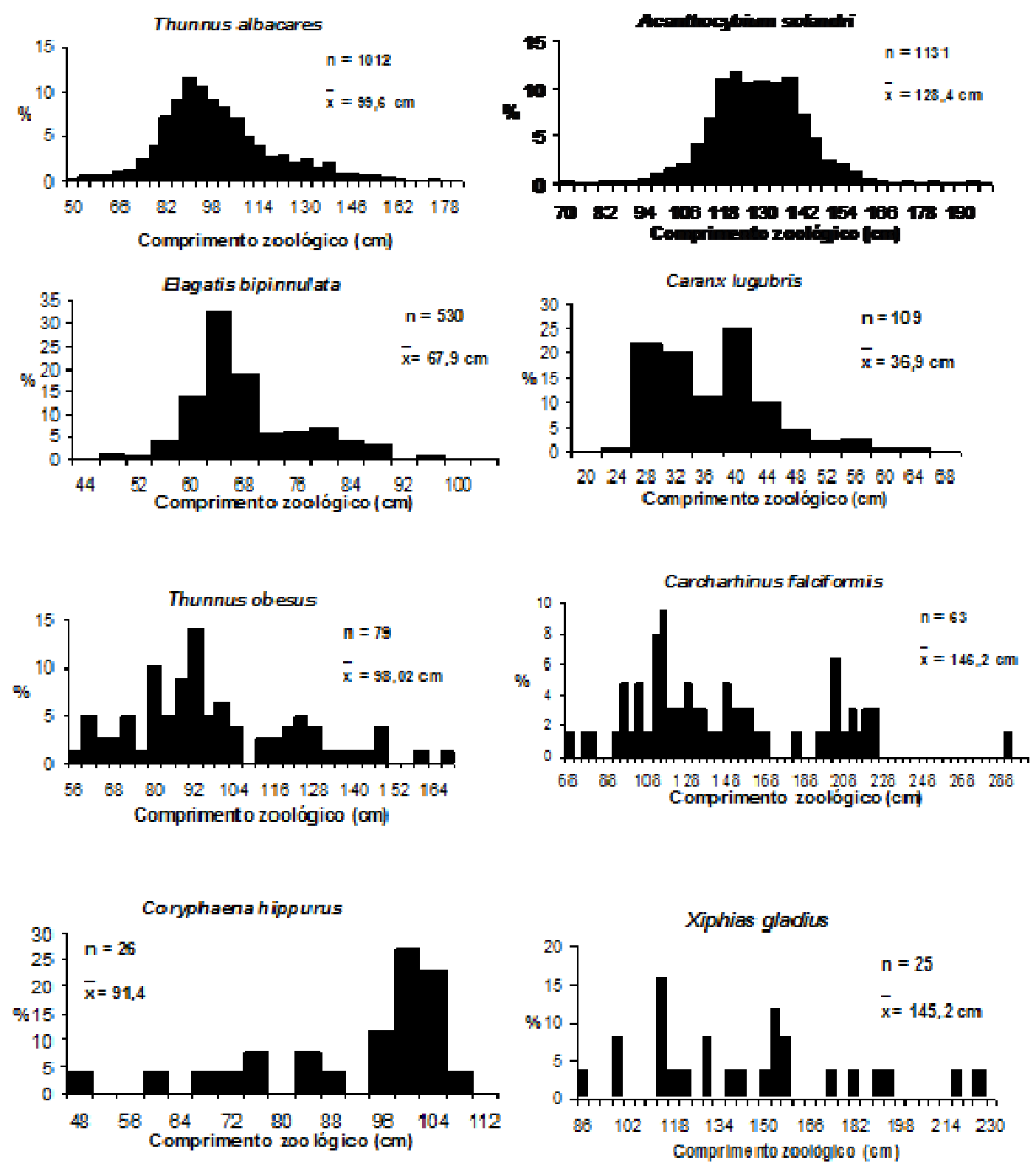

Figura 2 - Distribuições de comprimento, para ambos os sexos, das espécies mais capturadas no Arquipélago de São Pedro e São Paulo 
VASKE Jr et al. A pesca comercial de peixes pelágicos no arquipélago de São Pedro e São Paulo, Brasil.
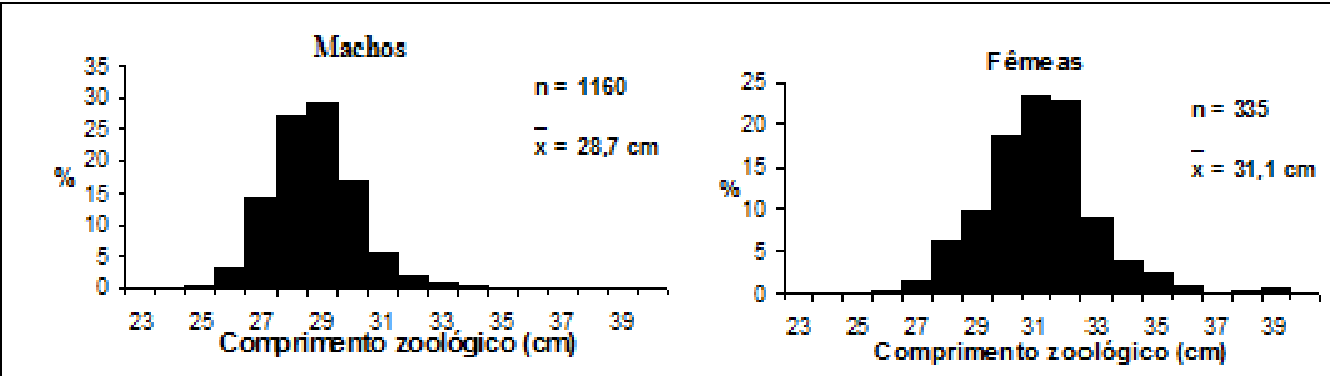

Figura 3 - Distribuições de comprimento para machos e fêmeas de voador-holandês no Arquipélago de São Pedro e São Paulo.

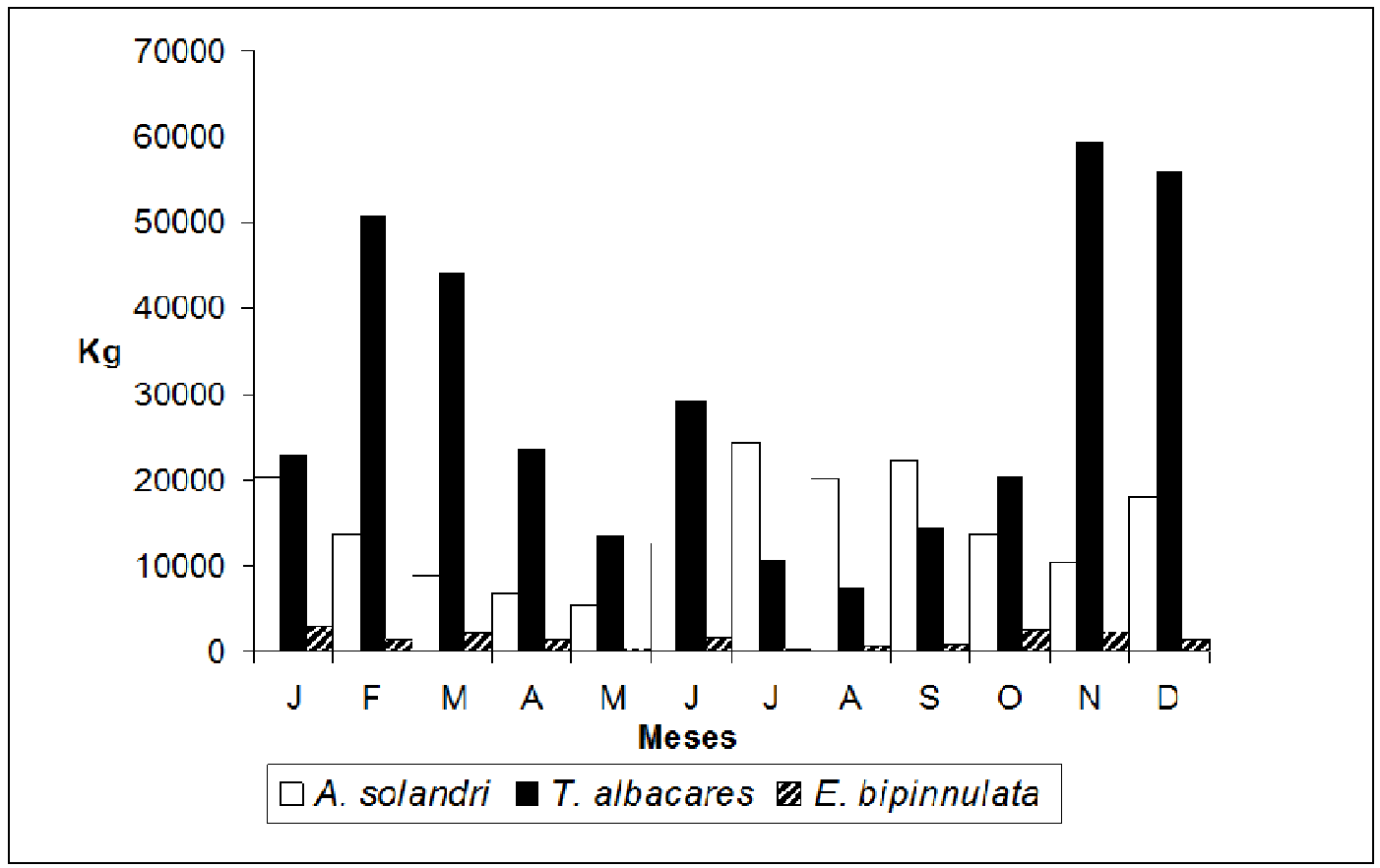

Figura 4 - Capturas mensais acumuladas de Acanthocybium solandri, Thunnus albacares, e Elagatis bipinnulata, entre 1998 e 2004 no Arquipélago de São Pedro e São Paulo. Fonte: Nortepesca-Natal. 